migraines, but only $24 \%$ offered any support. Migraine had negative impacts on relationships.

Conclusion The Migraine Voice Survey highlights the true burden of migraine in Australia and limitations of current management options.

\section{GEMCITABINE-RELATED RADIATION RECALL AS A CAUSE OF FOCAL MYOSITIS AND MUSCLE NECROSIS}

Sean Byrnes*. Neurology, The Canberra Hospital, Garran, ACT, Australia

10.1136/jnnp-2019-anzan.109

Introduction Radiation recall is a phenomenon in which chemotherapy triggers an inflammatory response in tissue previously subjected to radiation therapy. A wide variety of agents have been implicated. Cutaneous tissue is most frequently affected but other tissue can be involved; myositis has been associated with administration of gemcitabine in particular. Incidence has been estimated at less than $6 \%$ and the pathophysiology is not understood. We present a case report from Gosford Hospital, with the additional feature of positive SRP antibodies.

Case A 74 year old female presented with a one day history of left hip pain and inability to weight bear. She had been diagnosed with metastatic squamous cell carcinoma of the lung five months earlier and underwent palliative radiotherapy to a left acetabular metastasis. 12 days prior to presentation she completed her second cycle of chemotherapy with carboplatin and gemcitabine. Pre- and post-contrast CT and MRI demonstrated necrosis in left sartorius, with foci of myositis in other muscles of the thigh, and surrounding soft tissue oedema. Symptoms improved after chemotherapy was ceased. Myositis antibody studies subsequently revealed low level positive $\mathrm{Ku}$ and SRP antibodies.

Conclusion Radiation recall should be considered in the differential diagnosis of myositis in oncology patients. The serum of our patient contained SRP antibodies, which are associated with immune mediated necrotising myopathy. A previous case study reported gemcitabine-induced radiation recall muscle necrosis associated with dermatomyositis. These findings hint that radiation recall myositis may occur in the setting of a predisposition to immune mediated myopathy.

\section{CORRELATING STRUCTURE AND FUNCTION TO BETTER IDENTIFY SURROGATE END POINTS FOR CLINICAL TRIAL DESIGN: A LONGITUDINAL CLINICAL AND IMAGING STUDY OF PRIMARY PROGRESSIVE APHASIA}

Colin Mahoney*. University of Sydney, Camperdown, NSW, Australia

\subsection{6/jnnp-2019-anzan. 110}

Introduction Measuring longitudinal change in white matter tracts offers a highly sensitive way of monitoring the course of a range of neurodegenerative conditions. However, it remains unclear how structural changes correlate with symptom progression. Clinically meaningful outcomes remain a key requirement in therapeutic trial design so imaging biomarkers need to accurately predict these outcomes. Identifying surrogate clinical end points is of particular importance in neurodegenerative conditions where clinical change evolves slowly. To address this the current study aims to identify potential surrogate end points by assessing correlations between clinical and neuroimaging measures.

Methods 30 patients meeting consensus criteria for a diagnosis of primary progressive aphasia underwent longitudinal imaging and neuropsychological assessments at baseline and one year. A mixed effects model was designed to test for significant interactions over time between changes in neuropsychological performance and Fractional Anisotropy (FA) in key white matter tracts.

Results Declining single word comprehension correlated with reducing FA within bilateral inferior longitudinal fasciculus (ILF), bilateral superior longitudinal fasciculus (SLF) and the genu of the corpus callosum; declining naming ability correlated with reducing FA in the left ILF, right uncinate fasciculus and right SLF; declining word repetition correlated with reducing FA within the left ILF.

Conclusions Declining neuropsychological scores correlated with longitudinal decline in FA in a number of white matter tracts across an anatomically distributed language network. Correlations between function and structure provide evidence that monitoring structural white matter changes in the tracks identified may have value as a surrogate end point for future clinical trials.

\section{CHARACTERISING SLEEP AND FATIGUE IN PATIENTS WITH PRIMARY MITOCHONDRIAL DISEASE}

${ }^{2,1}$ Christine Wools, ${ }^{3}$ Carolyn Sue*, ${ }^{4}$ Peter Cistulli, ${ }^{5}$ Ryan Davis. ${ }^{1}$ Calvary healthcare Bethlehem Hospital, Parkdale, VIC, Australia; ${ }^{2}$ Royal Melbourne Hospital, Parkville, VIC, Australia; ${ }^{3}$ Neurogenetics, Royal North Shore Hospital, St Leonards, NSW, Australia; ${ }^{4}$ Respiratory medicine, Royal North Shore Hospital, St Leonards, NSW, Australia; ${ }^{5}$ Neurogenetics, Kolling Institute, St Leonards, NSW, Australia

10.1136/jnnp-2019-anzan. 111

Introduction Fatigue is common in patients with primary mitochondrial disease (PMD). There has been little prospective research into sleep pathology in these patients and assessment of scontributory factors to fatigue.

Methods Patients with PMD were prospectively assessed with overnight polysomnography in addition to measures of fatigue, muscle fatiguability, disease severity, sleep propensity and depression.

Results 16 patients participated, 15 completing inpatient polysomnography. Obstructive sleep apnoea (OSA) was common (53\%), affecting $5 / 9$ females $(56 \%)$ and $3 / 6$ males (50\%), although most cases were mild in severity. There was a trend to higher incidence of OSA in older patients but not other traditional risk factors, nor presence of myopathy. The Epworth Sleepiness Scale (ESS) was the best predictor of OSA, although not reaching significance.

Fatigue was common, with $81 \%$ of patients having significant fatigue on the Fatigue Severity score and $69 \%$ on the Fatigue Impact Scale. The two scores correlated well ( $r$ $=0.85, \mathrm{p} 0.01$ ). 